TRANSACTIONS OF THE

AMERICAN MATHEMATICAL SOCIETY

Volume 364, Number 8, August 2012, Pages 4041-4051

S 0002-9947(2012)05409-2

Article electronically published on February 17, 2012

\title{
EQUIVARIANT CHOW COHOMOLOGY OF NONSIMPLICIAL TORIC VARIETIES
}

\author{
HAL SCHENCK
}

\begin{abstract}
For a toric variety $X_{\Sigma}$ determined by a polyhedral fan $\Sigma \subseteq N$, Payne shows that the equivariant Chow cohomology is the $\operatorname{Sym}(N)$-algebra $C^{0}(\Sigma)$ of integral piecewise polynomial functions on $\Sigma$. We use the CartanEilenberg spectral sequence to analyze the associated reflexive sheaf $\mathcal{C}^{0}(\Sigma)$ on $\mathbb{P}_{\mathbb{Q}}(N)$, showing that the Chern classes depend on subtle geometry of $\Sigma$ and giving criteria for the splitting of $\mathcal{C}^{0}(\Sigma)$ as a sum of line bundles. For certain fans associated to the reflection arrangement $A_{n}$, we describe a connection between $C^{0}(\Sigma)$ and logarithmic vector fields tangent to $\mathrm{A}_{\mathrm{n}}$.
\end{abstract}

\section{INTRODUCTION}

In [1, Bifet, De Concini, Procesi show that the integral equivariant cohomology ring $H_{T}^{*}\left(X_{\Sigma}\right)$ of a smooth toric variety $X_{\Sigma}$ is isomorphic to the integral StanleyReisner ring $A_{\Sigma}$ of the unimodular fan $\Sigma$, and in [5], Brion shows that for $\Sigma$ simplicial, the rational equivariant Chow ring $A_{T}^{*}\left(X_{\Sigma}\right)_{\mathbb{Q}}$ is isomorphic to the ring of rational piecewise polynomial functions $C^{0}(\Sigma)_{\mathbb{Q}}$. A result of Billera 2 shows that for a simplicial fan, $C^{0}(\Sigma)_{\mathbb{Q}}$ is isomorphic to the rational Stanley-Reisner ring of the fan, so Brion's result is similar in spirit to 11. Brion and Vergne completed the picture for the simplicial case by showing in [6] that

$$
A_{T}^{*}\left(X_{\Sigma}\right)_{\mathbb{Q}} \simeq H_{T}^{*}\left(X_{\Sigma}\right)_{\mathbb{Q}} .
$$

Integral cohomology is more delicate; in 9 Payne exhibits a complete toric surface with 2-torsion in $H_{T}^{3}$. For a nonsimplicial fan $\Sigma$ there is no Stanley-Reisner ring, but the results of Billera and Brion suggest that $C^{0}(\Sigma)$ could serve as a possible substitute. In [9] Payne proves that the integral equivariant Chow ring $A_{T}^{*}\left(X_{\Sigma}\right)$ does indeed satisfy

$$
A_{T}^{*}\left(X_{\Sigma}\right) \simeq C^{0}(\Sigma) .
$$

We analyze the $S=\operatorname{Sym}_{\mathbb{Q}}(N)$-module structure of $C^{0}(\Sigma)_{\mathbb{Q}}$ and the associated reflexive sheaf $\mathcal{C}^{0}(\Sigma)$ on $\mathbb{P}_{\mathbb{Q}}(N)$. In contrast to the simplicial case, where $C^{0}(\Sigma)_{\mathbb{Q}}$ is completely determined by the combinatorics of $\Sigma$, in the nonsimplicial case there are surprising and subtle contributions from the geometry of $\Sigma$. We begin by defining a cellular chain complex $\mathcal{C}$ whose top homology module is $C^{0}(\Sigma)$, then study the lower homology modules of the complex. We give a complete description of the loci of the top dimensional support of the modules $H_{i}(\mathcal{C})$. Using a Cartan-Eilenberg spectral sequence, we also obtain sufficient criteria for the splitting of $\mathcal{C}^{0}(\Sigma)$ as a sum of line bundles.

Received by the editors March 1, 2010 and, in revised form, June 29, 2010.

2010 Mathematics Subject Classification. Primary 14M25; Secondary 14F43, 13D02, 52B99.

Key words and phrases. Chow ring, toric variety, piecewise polynomial function.

The author was supported by NSF 0707667.

(C)2012 American Mathematical Society Reverts to public domain 28 years from publication 


\subsection{Main results.}

Theorem 1. For all $i \geq 1, H_{d-i}(\mathcal{C})$ is supported in codimension at least $i+1$, and all associated primes of codimension $i+1$ are linear.

In Theorem 2.6 we give a combinatorial description of the codimension $i+1$ associated primes of $H_{d-i}(\mathcal{C})$ in terms of $\Sigma_{d-i}$ and $\Sigma_{d-i+1}$.

Theorem 2. If $H_{i}(\mathcal{C})$ is either Cohen-Macaulay of codimension $d-i+1$ or zero for all $i<d$, then $C^{0}(\Sigma)$ is free.

In particular, $C^{0}(\Sigma)$ can be free even when some lower homology modules are nonzero, whereas in the simplicial case, $C^{0}(\Sigma)$ is free iff $H_{d-i}(\mathcal{C})=0$ for all $i \geq 1$.

Example 1.1 ([8]). Let $\Sigma$ be the polyhedral fan obtained by coning over the complex below:

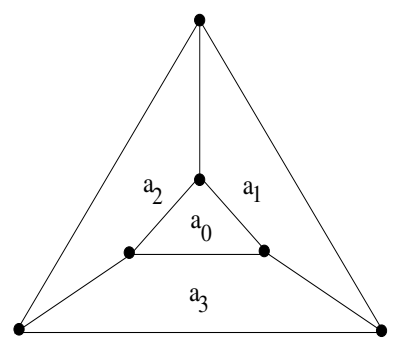

Figure 1. A two-dimensional section of $\Sigma$

The fan $\Sigma$ defines a three-dimensional toric variety, having four maximal cones, and a computation shows that the Hilbert polynomial of $C^{0}(\Sigma)$ is

$$
H P\left(C^{0}(\Sigma), k\right)=2 k^{2}+2 .
$$

The fan $\Sigma$ is nongeneric; in Figure 1 the three lines connecting boundary vertices to interior vertices meet at a point. Perturbing one vertex in Figure 1 so the symmetry is broken yields a combinatorially equivalent fan $\Sigma^{\prime}$, with

$$
H P\left(C^{0}\left(\Sigma^{\prime}\right), k\right)=2 k^{2}+1 .
$$

Both of these calculations follow from Corollary 2.9. The six interior facets of $\Sigma$ correspond to six lines in $\mathbb{P}^{2}$ defining the braid arrangement $\mathrm{A}_{3}$. Theorem 4.6 provides a second interpretation of the Hilbert polynomial.

In Example 2.10, we use Theorem 1 to analyze the four-dimensional analog of Example 1.1, showing that the module $C^{0}(\Sigma)$ is free with generators in degrees $\{0,1,2,3,4\}$. Motivated by this, we define two families of fans associated to the reflection arrangement $A_{n}$. One family corresponds to projective space, so the fans are all smooth and complete. The second family of fans generalizes the example above, and the fans are neither complete nor simplicial. We show:

Theorem 3. For both families of fans described above, $C^{0}(\Sigma)$ is isomorphic to the module of logarithmic vector fields on the reflection arrangement $\mathrm{A}_{\mathrm{n}}$. 


\section{Cellular homology}

Let $\Sigma$ be a $d$-dimensional polyhedral fan embedded in $\mathbb{Q}^{d}$. We make the simplifying assumption that $\Sigma$ is hereditary; this means for every nonempty face $\sigma$ of $\Sigma$, the dual graph of the star of $\sigma$ is connected. Henceforth, we use fan in place of hereditary rational polyhedral fan embedded in $\mathbb{Q}^{d}$. A $C^{0}$-spline on $\Sigma$ is a piecewise polynomial function such that two polynomials supported on $d$-faces which share a common $(d-1)$-face $\tau$ meet continuously along that face. Let $\Sigma_{i}^{0}$ denote the set of interior $i$ faces of $\Sigma\left(\Sigma_{d}^{0}=\Sigma_{d}\right)$, and let $f_{i}^{0}=\left|\Sigma_{i}^{0}\right|$; for a face $\tau$ write $\bar{\tau}$ for the linear span. The set of all $C^{0}$-splines on $\Sigma$ is a graded module over both $\mathbb{Z}\left[x_{1}, \ldots, x_{d}\right]$ and $\mathbb{Q}\left[x_{1}, \ldots, x_{d}\right]$. In [3], Billera and Rose observe that the module of splines of smoothness $r$ arises as the kernel of a matrix; for $r=0$ their result shows that there is a graded exact sequence,

$$
\begin{aligned}
& 0 \longrightarrow C^{0}(\Sigma) \longrightarrow S^{f_{d}} \oplus S^{f_{d-1}^{0}}(-1) \stackrel{\phi}{\longrightarrow} S^{f_{d-1}^{0}} \longrightarrow N \longrightarrow 0, \\
& \text { where } \phi=\left[\partial_{d} \mid \begin{array}{lll}
l_{\tau_{1}} & & \\
& \ddots & \\
& & l_{\tau_{m}}
\end{array}\right] \text {. }
\end{aligned}
$$

Write $\left[\partial_{d} \mid D\right]$ for $\phi$. To describe $\partial_{d}$, note that the rows of $\partial_{d}$ are indexed by $\tau \in \Sigma_{d-1}^{0}$. If $a_{1}, a_{2}$ denote the $d$-faces adjacent to $\tau$, then in the row corresponding to $\tau$ the continuity condition means that the only nonzero entries occur in the columns corresponding to $a_{1}, a_{2}$, and are $\pm(+1,-1)$. When $\Sigma$ is simplicial, $\partial_{d}$ is the top boundary map in the (relative) chain complex. By equation (1), $C^{0}(\Sigma)$ is a second syzygy, so the associated sheaf is a reflexive $\mathcal{O}_{\mathbb{P}^{d-1}}$-module, and by the Hirzebruch-Riemann-Roch theorem the coefficients of $\operatorname{HP}\left(C^{0}(\Sigma), k\right)$ yield the Chern classes of $C^{0}(\Sigma)$. Since

$$
N \simeq\left(\bigoplus_{\tau \in \Sigma_{d-1}^{0}} S / I_{\tau}\right) / \partial_{d}
$$

this suggests constructing a chain complex. For each $k$-dimensional face $\xi \prec \Sigma$, let $I_{\xi}$ be the ideal of $\bar{\xi}$. In [10], a chain complex $\mathcal{C}(\Delta)$ is defined for a simplicial fan $\Delta$, such that the top homology module of $\mathcal{C}(\Delta)$ computes splines of a fixed order of smoothness on $\Delta$. Using cellular homology, we next carry out a similar construction for polyhedral $\Sigma$.

Definition 2.1. For a polyhedral fan $\Sigma$, let $\mathcal{C}$ be the complex of $S=\mathbb{Q}\left[x_{1}, \ldots, x_{d}\right]$ modules, with cellular differential $\partial_{i}$.

$$
0 \longrightarrow \bigoplus_{\sigma \in \Sigma_{d}} S \stackrel{\partial_{d}}{\longrightarrow} \bigoplus_{\tau \in \Sigma_{d-1}^{0}} S / I_{\tau} \stackrel{\partial_{d-1}}{\longrightarrow} \bigoplus_{\psi \in \Sigma_{d-2}^{0}} S / I_{\psi} \stackrel{\partial_{d-2}}{\longrightarrow} \ldots \stackrel{\partial_{2}}{\longrightarrow} \bigoplus_{v \in \Sigma_{1}^{0}} S / I_{v} \longrightarrow 0
$$

By construction, $H_{d}(\mathcal{C})=C^{0}(\Sigma)$, and taking Euler characteristics yields

\section{Proposition 2.2.}

$$
H P\left(C^{0}(\Sigma), k\right)=\sum_{i=1}^{d}(-1)^{d-i} f_{i}^{0} \cdot\left(\begin{array}{c}
k+i-1 \\
i-1
\end{array}\right)+\sum_{i=1}^{d-1}(-1)^{d-1-i} H P\left(H_{i}(\mathcal{C}), k\right) .
$$

Lemma 2.3. For $i \geq 1, H_{d-i}(\mathcal{C})$ is supported in codimension at least $i+1$. 
Proof. Since

$$
H_{d-i}(\mathcal{C})=H\left(\bigoplus_{\sigma \in \Sigma_{d-i+1}} S / I_{\sigma} \stackrel{\partial_{d-i+1}}{\longrightarrow} \bigoplus_{\tau \in \Sigma_{d-i}^{0}} S / I_{\tau} \stackrel{\partial_{d-i}}{\longrightarrow} \bigoplus_{\psi \in \Sigma_{d-i-1}^{0}} S / I_{\psi}\right),
$$

localizing at a prime $P$ of codimension $i$ forces the vanishing of the middle term, unless $P=I_{\tau}$. But in this case, there exists $\sigma \in \Sigma_{d-i+1}^{0}$ with $\tau \subseteq \sigma$, and so the localized map $\partial_{d-i+1}$ is onto: though there can be several distinct $\tau_{i} \in \Sigma_{d-i}^{0}$ with $\overline{\tau_{i}}=V(P)$, convexity implies that a $d-i+1$ face $\sigma$ containing one such $\tau_{i}$ cannot contain any others. Thus $H_{d-i}(\mathcal{C})_{P}=0$ if $\operatorname{codim}(P) \leq i$.

Lemma 2.4. All associated primes of $H_{d-i}(\mathcal{C})$ of codimension $i+1$ are linear.

Proof. Let $P$ be an associated prime of codimension $i+1$, minimally generated by $\left\langle f_{1}, \ldots, f_{m}\right\rangle$. If less than $i+1$ of the $f_{j}$ are linear, then Lemma 2.3 shows

$$
\left(\bigoplus_{\tau \in \Sigma_{d-i}^{0}} S / I_{\tau}\right)_{P}=0
$$

unless $P$ contains exactly $i$ linear forms such that $\left\langle f_{1}, \ldots, f_{i}\right\rangle=I_{\tau}$, and in this case, the localization of $\partial_{d-i+1}$ is surjective. So if $H_{d-i}(\mathcal{C})_{P} \neq 0$, then $P$ must have at least $i+1$ minimal generators which are linear. Since $\operatorname{codim}(P)=i+1$, $P=\left\langle l_{1}, \ldots, l_{i+1}\right\rangle$.

Definition 2.5. Let $\Sigma$ be a $d$-dimensional fan, and $\xi$ a codim $(i+1)$ linear subspace. Define a graph $G_{\xi}(\Sigma)$, with a vertex $v_{\tau}$ for every $\tau \in \Sigma_{d-i}^{0}$ with $\xi \in \bar{\tau}$ and an edge $\overline{v_{\tau} v_{\sigma}}$ if there exists $\gamma \in \Sigma_{d-i+1}^{0}$ with $\partial_{d-i+1}(\gamma)=\tau \pm \sigma+\cdots$ and $\xi=\bar{\tau} \cap \bar{\sigma}$.

Theorem 2.6. For $\xi$ a linear subspace of codimension $i+1, I_{\xi}$ is an associated prime of $H_{d-i}(\mathcal{C})$ iff $G_{\xi}(\Sigma)=\amalg \Delta_{j}$ has a component $\Delta_{j}$ such that the following two conditions hold:

(1) $\Delta_{j}$ does not correspond to a loop around $\tau \in \Sigma_{d-i-1}^{0}$.

(2) $\Delta_{j}$ has no vertex of valence one.

Proof. Localize $\mathcal{C}$ at $I_{\xi}$, and write the localized complex as $\mathcal{C}_{\xi}$.

Case 1. Suppose some (possibly several) $\tau \in \Sigma_{d-i-1}^{0}$ have $\tau \in \xi$. In the localized complex, we have

$$
\cdots \stackrel{\partial_{d-i+1}}{\longrightarrow} \bigoplus_{\substack{\xi \prec \bar{\psi} \\ \psi \in \Sigma_{d-i}^{0}}}\left(S / I_{\psi}\right)_{I_{\xi}} \stackrel{\partial_{d-i}}{\longrightarrow} \bigoplus_{\substack{\xi=\bar{\tau} \\ \tau \in \Sigma_{d-i-1}^{0}}}\left(S / I_{\tau}\right)_{I_{\xi}} \longrightarrow 0 .
$$

A codimension $i$ face $\psi$ can contain at most one facet $\tau$ of the form above, and choosing any such facet shows that (in the localization) $\partial_{d-i}$ is surjective. This yields

$$
0 \longrightarrow H_{d-i}\left(\mathcal{C}_{\xi}\right) \longrightarrow\left(\bigoplus_{\substack{\xi \prec \bar{\psi} \\ \psi \in \Sigma_{d-i}^{0}}}\left(S / I_{\psi}\right)_{I_{\xi}}\right) / \operatorname{im}\left(\partial_{d-i+1}\right) \stackrel{\partial_{d-i}}{\longrightarrow} \bigoplus_{\substack{\xi=\bar{\tau} \\ \tau \in \Sigma_{d-i-1}^{0}}}\left(S / I_{\tau}\right)_{I_{\xi}} \longrightarrow 0 .
$$

The map $\partial_{d-i}$ splits in $\mathcal{C}_{\xi}$. To see this, note that in $\mathcal{C}_{\xi}$, two types of codimension $i$ face $\psi$ can appear: those which actually have a $\operatorname{codim}(i+1)$ face $\tau$ lying in $\xi$, and those which do not. In the first situation, fix $\tau \in \Sigma_{d-i-1}^{0}$ such that $\tau \in \xi$. By convexity, there exists a codimension $i-1$ face $\gamma$ with 
$\partial_{d-i+1}(\gamma)=\psi_{1} \pm \psi_{2} \pm \cdots$, and $\tau=\psi_{1} \cap \psi_{2}$. Looping around $\tau$ yields an isomorphism $\bigoplus_{\tau \in \psi \in \Sigma_{d-i}^{0}}\left(S / I_{\psi}\right)_{I_{\xi}} / \operatorname{im}\left(\partial_{d-i+1}\right) \simeq\left(S / I_{\tau}\right)_{I_{\xi}}$, and $H_{d-i}\left(\mathcal{C}_{\xi}\right)$ vanishes. In the latter situation, the localized map $\partial_{d-i}$ is zero, so

$$
H_{d-i}\left(\mathcal{C}_{\xi}\right) \simeq \bigoplus_{\substack{\xi \prec \bar{\psi}, \psi \in \Sigma_{d-i}^{0} \\ \xi \neq \bar{\tau} \mid \tau \in \partial(\psi)}}\left(S / I_{\psi}\right)_{I_{\xi}} / \operatorname{im}\left(\partial_{d-i+1}\right) .
$$

Case 2. No $\tau \in \Sigma_{d-i-1}$ satisfies $\tau \in \xi$. Since no $\tau \in \xi$,

$$
\left(\bigoplus_{\tau \in \Sigma_{d-i-1}^{0}} S / I_{\tau}\right)_{I_{\xi}}=0
$$

Hence, $H_{d-i}\left(\mathcal{C}_{\xi}\right)$ is as in equation (2). By convexity, a codimension $i-1$ face $\gamma$ can have at most two facets $\psi_{i}$ with $\xi \prec \bar{\psi}_{i}$. Thus, the localized map $\partial_{d-i+1}$ will have columns with either one or two nonzero entries. In the former case, the corresponding generator of $S / I_{\psi}$ is killed by $\partial_{d-i+1}$. In the latter case, quotienting by a column with two nonzero entries gives a cokernel of the form $S / I_{\psi_{1}}+I_{\psi_{2}}$, where $\psi_{1}$ and $\psi_{2}$ are the two codimension $i$ faces with $\xi \prec \overline{\psi_{i}}$. Thus, $I_{\psi_{1}}+I_{\psi_{2}}=I_{\xi}$. Write $G_{\xi}(\Sigma)=\amalg \Delta_{j}$. If $\Delta_{i}$ has a vertex with valence one, this means the localized map $\partial_{d-i+1}$ has column with single nonzero entry. Let $a_{\xi}$ denote the number of $\Delta_{i}$ with no vertex of valence one. We have shown $H_{d-i}\left(\mathcal{C}_{\xi}\right) \simeq\left(S / I_{\xi}\right)_{I_{\xi}}^{a_{\xi}}$, and the result follows.

Corollary 2.7. Let $L_{i}$ denote the Grassmannian of $\operatorname{codim}(i)$ linear subspaces. Associate to a point of $L_{i}$ the corresponding subspace, let $a_{\xi}$ be as above, and

$$
\alpha_{i}=\sum_{\xi \in L_{i+1}} a_{\xi}
$$

Then

$$
H P\left(H_{d-i}(\mathcal{C}), k\right)=\alpha_{i} \cdot\left(\begin{array}{c}
k+d-i-2 \\
d-i-2
\end{array}\right)+O\left(k^{d-i-3}\right) .
$$

Corollary 2.8. $\alpha_{1}=\sum_{\xi \in L_{2}} \operatorname{rank} H_{1}\left(G_{\xi}(\Sigma), \mathbb{Z}\right)-f_{d-2}^{0}$.

Proof. For $\xi \in L_{2}$, the components $\Delta_{i}$ are homeomorphic to circles or segments.

Corollary 2.9. For a fan $\Sigma \in \mathbb{Z}^{3}$,

$$
H P\left(C^{0}(\Sigma), k\right)=f_{3} \cdot\left(\begin{array}{c}
k+2 \\
2
\end{array}\right)-f_{2}^{0}(k+1)+f_{1}^{0}+\alpha_{1}
$$

Example 2.10. Consider a four-dimensional version of Example 1.1 place a small regular tetrahedron $T$ symmetrically inside a large regular tetrahedron $T^{\prime}$, and connect corresponding vertices. The maximal faces are the convex hulls of the corresponding facets of $T$ and $T^{\prime} . C^{0}(\Sigma)$ is a free $S$-module, with generators in degrees $\{0,1,2,3,4\}$, so the Hilbert polynomial is

$$
\sum_{i=0}^{4}\left(\begin{array}{c}
k+3-i \\
3
\end{array}\right)=5 \cdot\left(\begin{array}{c}
k+3 \\
3
\end{array}\right)-10\left(\begin{array}{c}
k+2 \\
2
\end{array}\right)+10\left(\begin{array}{c}
k+1 \\
1
\end{array}\right)-5 .
$$

If $v$ is the central point of symmetry, $G_{v}(\Sigma)$ is the 1-skeleton of a tetrahedron, so $H P\left(H_{2}(\mathcal{C}), k\right)=H P\left(S / I_{v}, k\right)=1$. Since $f_{0}=4$, this yields the expected constant term in the Hilbert polynomial. 


\section{Cartan-Eilenberg spectral Sequence}

Write the complex $\mathcal{C}$ in the first quadrant, with $\mathcal{C}_{i}$ appearing in position $(i, 0)$. Taking a Cartan-Eilenberg resolution for $\mathcal{C}$ and applying the functor $\operatorname{Hom}_{S}(\bullet, S)$ yields a first quadrant double complex. For the vertical filtration, ${ }_{v} \mathrm{E}_{i j}^{1}$ is $\operatorname{Ext}_{S}^{j}\left(\mathcal{C}_{i}, S\right)$, so since the $S / I_{\tau}$ are complete intersections,

$$
{ }_{v} \mathrm{E}_{i j}^{1}= \begin{cases}\bigoplus_{\tau \in \Sigma_{i}^{0}} S(i) / I_{\tau} & \text { if } j=d-i ; \\ 0 & \text { otherwise. }\end{cases}
$$

Thus ${ }_{v} \mathrm{E}_{i j}^{1}={ }_{v} \mathrm{E}_{i j}^{\infty}$. Write $G_{i j}$ for the $j^{\text {th }}$ module in a free resolution for $H_{i}(C)$. From the Cartan-Eilenberg construction, it follows that for the horizontal filtration

$$
{ }_{h} \mathrm{E}_{i j}^{1}=\operatorname{Hom}_{S}\left(G_{i j}, S\right) .
$$

Thus,

$$
h \mathrm{E}_{i j}^{2}=\operatorname{Ext}_{S}^{j}\left(H_{i}(\mathcal{C}), S\right) .
$$

Example 3.1. Let $d=4$. Then the ${ }_{v} \mathrm{E}_{i, j}^{1}$ terms are:

\begin{tabular}{|l|c|c|c|c|c|}
\hline & $i=0$ & 1 & 2 & 3 & 4 \\
\hline$j=3$ & & $\bigoplus_{v \in \Sigma_{1}^{0}} S / I_{v}$ & & & \\
\hline$j=2$ & & & $\bigoplus_{\tau \in \Sigma_{2}^{0}} S / I_{\psi}$ & & \\
\hline$j=1$ & & & & $\bigoplus_{\tau \in \Sigma_{3}^{0}} S / I_{\tau}$ & \\
\hline$j=0$ & & & & & $\bigoplus_{\sigma \in \Sigma_{4}} S$ \\
\hline
\end{tabular}

\begin{tabular}{|c|c|c|c|c|c|}
\hline & $i=0$ & 1 & 2 & 3 & 4 \\
\hline$j=4$ & & $\operatorname{Ext}^{4}\left(H_{1}(\mathcal{C}), S\right)$ & $\operatorname{Ext}^{4}\left(H_{2}(\mathcal{C}), S\right)$ & $\operatorname{Ext}^{4}\left(H_{3}(\mathcal{C}), S\right)$ & $\operatorname{Ext}^{4}\left(C^{0}(\Sigma), S\right)$ \\
\hline$j=3$ & & & $\operatorname{Ext}^{3}\left(H_{2}(\mathcal{C}), S\right)$ & $\operatorname{Ext}^{3}\left(H_{3}(\mathcal{C}), S\right)$ & $\operatorname{Ext}^{3}\left(C^{0}(\Sigma), S\right)$ \\
\hline$j=2$ & & & & $\operatorname{Ext}^{2}\left(H_{3}(\mathcal{C}), S\right)$ & $\operatorname{Ext}^{2}\left(C^{0}(\Sigma), S\right)$ \\
\hline$j=1$ & & & & & $\operatorname{Ext}^{1}\left(C^{0}(\Sigma), S\right)$ \\
\hline$j=0$ & & & & & $C^{0}(\Sigma)^{\vee}$ \\
\hline
\end{tabular}

By Lemma 2.3, for $i \geq 1, H_{d-i}(\mathcal{C})$ is supported in codimension at least $i+1$, so for the horizontal filtration, the ${ }_{h} \mathrm{E}_{i j}^{2}$ terms are:

Theorem 3.2. If $H_{i}(\mathcal{C})$ is Cohen-Macaulay with $\operatorname{codim}\left(H_{i}(\mathcal{C})\right)=d-i+1$ for all $i<d$, then $C^{0}(\Sigma)$ is free.

Proof. By local duality, $\operatorname{Ext}^{i}(M, S)$ vanishes if $i<\operatorname{codim}(M)$ and $i>\operatorname{pdim}(M)$. The Cohen-Macaulay condition implies $\operatorname{codim}(M)=\operatorname{pdim}(M)$, which combined with the assumption that $\operatorname{codim}\left(H_{i}(\mathcal{C})\right)=d-i+1$ implies that

$$
{ }_{h} \mathrm{E}_{i j}^{2}= \begin{cases}\operatorname{Ext}^{j}\left(H_{i}(\mathcal{C}), S\right) & \text { if } j+i=d+1 ; \\ 0 & \text { otherwise. }\end{cases}
$$

Thus, the $d_{2}$ and higher differentials from the terms ${ }_{h} \mathrm{E}_{d j}^{2}$ must all vanish. Comparing to the vertical filtration shows that $H_{i}$ (Tot) is concentrated in degree $d$, so that ${ }_{h} \mathrm{E}_{d j}^{2}=\operatorname{Ext}^{j}\left(C^{0}(\Sigma), S\right)=0$ for $j>0$. Note that the condition $\operatorname{codim}\left(H_{i}(\mathcal{C})\right)=$ $d-i+1$ can be weakened to include the case $H_{i}(\mathcal{C})=0$. 
Corollary 3.3. If $H_{i}(\mathcal{C})$ is Cohen-Macaulay with either $\operatorname{codim}\left(H_{i}(\mathcal{C})\right)=d-i+1$ or $H_{i}(\mathcal{C})=0$ for all $i<d$, then (letting $\alpha_{0}=\alpha_{-1}=0$ )

$$
H P\left(C^{0}(\Sigma), k\right)=\sum_{i=1}^{d}(-1)^{d-i}\left(f_{i}^{0}+\alpha_{d-1-i}\right) \cdot\left(\begin{array}{c}
k+i-1 \\
i-1
\end{array}\right) .
$$

When $\Sigma$ is simplicial, Lemma 2.3 can be strengthened [10] to show that for $i \geq 1$, $H_{d-i}(\mathcal{C})$ is supported in codimension at least $i+2$, and that

$$
C^{0}(\Sigma) \text { is free iff } H_{i}(\mathcal{C})=0 \text { for all } i<d .
$$

In Example 1.1, $C^{0}(\Sigma)$ and $C^{0}\left(\Sigma^{\prime}\right)$ are both free; for $\Sigma^{\prime}$ all the lower homology modules of $\mathcal{C}$ vanish, while for $\Sigma, H_{2}(\mathcal{C}) \neq 0$. Even in the simplicial case, there are easy examples where $C^{0}(\Sigma)$ is nonfree.

Example 3.4. Consider the cone over the planar complex $\Sigma$ below, where the (cone over) the central triangle has been removed:

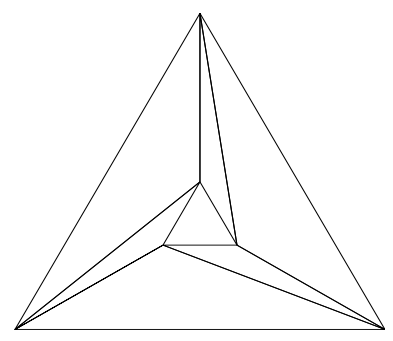

Figure 2. A simplicial nonfree $\Sigma$

The topological nontriviality of $\Sigma$ manifests in the nonvanishing of $H_{2}(\mathcal{C})$, which has high codimension: $\operatorname{Ext}^{2}\left(H_{2}(\mathcal{C})\right)=0$, but $\operatorname{Ext}^{3}\left(H_{2}(\mathcal{C})\right) \neq 0$. Comparing terms in the spectral sequence shows that this forces

$$
\left.\operatorname{Ext}^{1}\left(C^{0}(\Sigma), S\right)\right) \neq 0 \text {. }
$$

$C^{0}(\Sigma)$ is reflexive sheaf on $\mathbb{P}^{2}$, so is locally free. The above shows it does not split.

Definition 3.5. A pure dimensional polyhedral complex is shellable if there exists an ordering of facets $\left\{P_{1}, \ldots, P_{k}\right\}$ so that $\partial\left(P_{1}\right)$ is shellable, and $P_{i} \cap\left(\bigcup_{j<i} P_{j}\right)$ is the start of a shelling of $\partial\left(P_{i}\right)$

If $P$ is simplicial, an induction shows that shellability implies the Stanley-Reisner ring $A_{\Delta}$ is Cohen-Macaulay [7. For $\Sigma$ a pure polyhedral complex, if $\Sigma^{\prime}$ is obtained from $\Sigma$ by removing a full-dimensional cell $P$, then all faces of $P$ are part of the boundary of $\Sigma^{\prime}$. This gives a short exact sequence of complexes as in Definition 2.1. where $\mathcal{C}$ and $\mathcal{C}^{\prime}$ correspond to $\Sigma$ and $\Sigma^{\prime}$, and $\mathcal{C}^{\prime \prime}$ comes from $P$ and all its faces. This yields a long exact sequence.

$$
0 \longrightarrow\left\langle\prod_{\tau \in P_{d-1}} l_{\tau}\right\rangle \longrightarrow C^{0}(\Sigma) \longrightarrow C^{0}\left(\Sigma^{\prime}\right) \longrightarrow H_{d-1}\left(\mathcal{C}^{\prime \prime}\right) \longrightarrow \cdots .
$$

In the simplicial case, $H_{d-1}\left(\mathcal{C}^{\prime \prime}\right)=0$, but this need not hold in the polyhedral case. Nevertheless, computational evidence leads us to ask,

Question 3.6. If $\Sigma$ is shellable, is $C^{0}(\Sigma)$ a free $S$-module? 


\section{FAns Associated to TYPE A ROOT SYSTEMS}

In this section, we analyze two very different families of fans associated to the reflection arrangement $A_{n}$. Though the fans are quite different, for both families $C^{0}(\Sigma)$ is isomorphic to the module of vector fields tangent to $\mathrm{A}_{\mathrm{n}}$. Given a fan, we associate to it the hyperplane arrangement in $\mathbb{P}^{d-1}$ defined by $\bar{\tau}$ such that $\tau \in \Sigma_{d-1}^{0}$. For a collection of hyperplanes

$$
\mathcal{A}=\bigcup_{i=1}^{n} H_{i} \subseteq \mathbb{C}^{d}
$$

the intersection lattice $L(\mathcal{A})$ consists of the intersections of the elements of $\mathcal{A}$; the rank of $x \in L_{\mathcal{A}}$ is simply the codimension of $x . \mathbb{C}^{d}$ is the lattice element $\hat{0}$; the rank one elements are the hyperplanes themselves.

Definition 4.1. The Möbius function $\mu: L(\mathcal{A}) \longrightarrow \mathbb{Z}$ is defined by

$$
\begin{aligned}
& \mu(\hat{0})=1, \\
& \mu(t)=-\sum_{s<t} \mu(s), \quad \text { if } \hat{0}<t .
\end{aligned}
$$

The Poincaré polynomial is

$$
\pi(\mathcal{A}, t)=\sum_{x \in L(\mathcal{A})} \mu(x) \cdot(-t)^{\operatorname{rank}(x)} .
$$

One of the main algebraic objects associated to an arrangement is the graded $S$-module $D(\mathcal{A})$ of vector fields tangent to $\mathcal{A}$.

Definition 4.2. $D(\mathcal{A})=\left\{\theta \mid \theta\left(\alpha_{i}\right) \in\left\langle\alpha_{i}\right\rangle\right.$ for all $\left.V\left(\alpha_{i}\right) \in \mathcal{A}\right\} \subseteq \operatorname{Der}_{\mathbb{Q}}(S)$.

It follows immediately from the definition that $D(\mathcal{A})$ can be computed as the kernel of a matrix similar to $\phi$ in equation (1): $D(\mathcal{A})$ is the kernel of

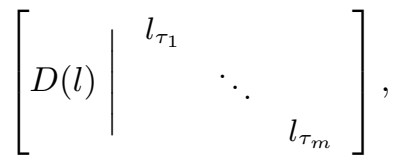

where $D(l)$ is an $|\mathcal{A}| \times d$ matrix. If $l_{j}=\sum a_{i}^{j} x_{i}$, then the $j$ th row of $D(l)$ is $\left[a_{1}^{j}, \ldots, a_{d}^{j}\right]$.

Definition 4.3. $\mathcal{A}$ is free if $D(\mathcal{A}) \simeq \bigoplus S\left(-d_{i}\right)$; the $d_{i}$ are the exponents of $\mathcal{A}$.

In 11, Terao proves that if $D(\mathcal{A}) \simeq \bigoplus S\left(-d_{i}\right)$, then $\pi(\mathcal{A}, t)=\prod\left(1+d_{i} t\right)$, and in 12 shows that if $G$ is a finite reflection group acting with no fixed points, then the arrangement of reflecting hyperplanes $\mathcal{A}_{G}$ is free, with $d_{i}$ equal to the degrees of generators of $S^{G}$.

Example 4.4. The reflecting hyperplanes of $S L(n)$ are $\bigcup_{1 \leq i<j \leq n+1} V\left(x_{j}-x_{i}\right)=$ $\mathrm{A}_{\mathrm{n}} \subseteq \mathbb{C}^{n+1}$. The symmetric group $S_{n}$ action fixes the subspace $(t, \ldots, t)$. Projecting along this subspace yields $\mathrm{A}_{\mathrm{n}} \subseteq \mathbb{C}^{n}$, with $S_{n}$ acting without fixed points. By $\left[12, \mathrm{~A}_{\mathrm{n}} \subseteq \mathbb{C}^{n}\right.$ is free with exponents $\{1,2, \ldots, n\}$ and $\mathrm{A}_{\mathrm{n}} \subseteq \mathbb{C}^{n+1}$ is free with exponents $\{0,1,2, \ldots, n\}$. Up to projective transformation, $A_{3}$ is exactly the configuration defined by the six $\tau \in \Sigma_{2}^{0}$ appearing in Example 1.1 The corresponding $\bar{\tau}$ yield an arrangement of six planes through the origin $\mathbb{C}^{3}$, depicted in Figure 3 as a set of lines in $\mathbb{P}^{2}$. 

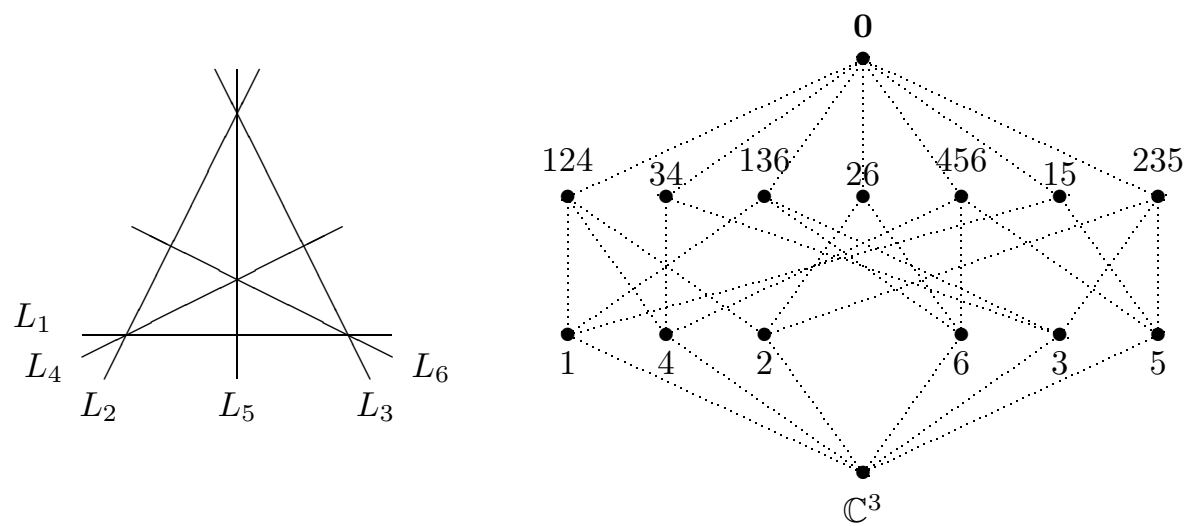

Figure 3 . The braid arrangement $A_{3}$ and its intersection lattice in $\mathbb{C}^{3}$

In Example 2.10, $C^{0}(\Sigma)$ is free, with generators in degrees $\{0,1,2,3,4\}$, and the associated arrangement is $\mathrm{A}_{4}$. This motivates:

Definition 4.5. Let $v_{i}=(n+1) \mathbf{e}_{\mathbf{i}}-\sum_{i=1}^{n} \mathbf{e}_{\mathbf{i}}$ and $\Delta_{2}=\left\{v_{1}, \ldots, v_{n}\right\}$. Define cones $a_{0}=$ cone $\left\{\mathbf{e}_{1}, \ldots, \mathbf{e}_{\mathbf{n}}\right\}$, and for $i \in\{1, \ldots, n\}, a_{i}=\operatorname{cone}\left\{\Delta_{2} \backslash v_{i}, a_{0} \backslash \mathbf{e}_{\mathbf{i}}\right\}$. Then $P_{2}\left(\mathrm{~A}_{\mathrm{n}}\right)=\left\{a_{0}, a_{1}, \ldots, a_{n}\right\}$ is a polyhedral fan, which is a subdivision of the simplex cone $S=\operatorname{cone}\left(\Delta_{2}\right)$.

$P_{2}\left(A_{3}\right)$ appears in Example 1.1 and $P_{2}\left(A_{4}\right)$ in Example 2.10,

Theorem 4.6. $C^{0}\left(P_{2}\left(\mathrm{~A}_{\mathrm{n}}\right)\right) \simeq D\left(\mathrm{~A}_{\mathrm{n}}\right)$.

Proof. The main point is that the passage from $n$ to $n+1$ induces the same modification in the matrices which compute $D\left(\mathrm{~A}_{\mathrm{n}}\right)$ and $C^{0}\left(P_{2}\left(\mathrm{~A}_{\mathrm{n}}\right)\right)$. A computation shows that the hyperplanes $\bar{\tau}$ for $\tau \in P\left(\mathrm{~A}_{\mathrm{n}}\right)_{d-1}^{0}$ are of the form $V\left(x_{i}\right)$ or $V\left(x_{j}-x_{i}\right)$ for $1 \leq i<j \leq n$. Order the $d$-faces of $P_{2}\left(\mathrm{~A}_{\mathrm{n}}\right)$ as $\left\{a_{d}, a_{d-1}, \ldots, a_{0}\right\}$ (see Figure 1), and order $\tau \in \Sigma_{d-1}^{0}$ so that the $x_{i}$ are the first $n$ elements, and the remaining hyperplanes form a second block. For example, the lines $L_{1}, L_{2}, L_{3}$ in Figure 3 correspond to the boundary of the inner triangle in Figure 1, and lines $L_{4}, L_{5}, L_{6}$ correspond to the $\tau \in \Sigma_{2}^{0}$ defining $V\left(x_{i}-x_{j}\right)$. Thus, the matrix which computes $C^{0}\left(P_{2}\left(\mathrm{~A}_{3}\right)\right)$ has the form

$$
\left[\begin{array}{cccccccccc}
-1 & 0 & 0 & 1 & x_{1} & 0 & 0 & 0 & 0 & 0 \\
0 & -1 & 0 & 1 & 0 & x_{2} & 0 & 0 & 0 & 0 \\
0 & 0 & -1 & 1 & 0 & 0 & x_{3} & 0 & 0 & 0 \\
-1 & 0 & 1 & 0 & 0 & 0 & 0 & x_{3}-x_{1} & 0 & 0 \\
0 & -1 & 1 & 0 & 0 & 0 & 0 & 0 & x_{3}-x_{2} & 0 \\
-1 & 1 & 0 & 0 & 0 & 0 & 0 & 0 & 0 & x_{2}-x_{1}
\end{array}\right] .
$$

If we consider $\mathrm{A}_{3} \subseteq \mathbb{C}^{4}$, and order so that the equations $x_{4}-x_{i}$ appear first, then the matrix which computes $D\left(\mathrm{~A}_{3}\right)$ has the form

$$
\left[\begin{array}{cccccccccc}
-1 & 0 & 0 & 1 & x_{4}-x_{1} & 0 & 0 & 0 & 0 & 0 \\
0 & -1 & 0 & 1 & 0 & x_{4}-x_{2} & 0 & 0 & 0 & 0 \\
0 & 0 & -1 & 1 & 0 & 0 & x_{4}-x_{3} & 0 & 0 & 0 \\
-1 & 0 & 1 & 0 & 0 & 0 & 0 & x_{3}-x_{1} & 0 & 0 \\
0 & -1 & 1 & 0 & 0 & 0 & 0 & 0 & x_{3}-x_{2} & 0 \\
-1 & 1 & 0 & 0 & 0 & 0 & 0 & 0 & 0 & x_{2}-x_{1}
\end{array}\right] .
$$


Since $\mathrm{A}_{3} \subseteq \mathbb{C}^{4}$ contains the subspace $(t, t, \ldots, t), D\left(\mathrm{~A}_{3}\right)$ has a free summand of degree zero. To apply [12], $G$ must act with no fixed points. Specializing to $x_{4}=0$ yields $\mathrm{A}_{3} \subseteq \mathbb{C}^{3}$ with this property. The general case follows by induction.

Corollary 4.7. As an $S$-module, $A_{T}^{*}\left(X_{P_{2}\left(\mathrm{~A}_{\mathrm{n}}\right)}\right) \mathbb{Q} \simeq \bigoplus_{i=0}^{n} S(-i)$.

Proposition 4.8. Let $P_{1}\left(\mathrm{~A}_{\mathrm{n}}\right)$ denote the fan such that $X_{P_{1}\left(\mathrm{~A}_{\mathrm{n}}\right)}=\mathbb{P}^{n}$. Then

$$
C^{0}\left(P_{1}\left(\mathrm{~A}_{\mathrm{n}}\right)\right) \simeq D\left(\mathrm{~A}_{\mathrm{n}}\right) .
$$

Proof. $P_{1}\left(\mathrm{~A}_{\mathrm{n}}\right)$ has defining hyperplanes $\mathrm{A}_{n}$, and $n+1$ top-dimensional cones; order the cones in the same way as for $P_{2}\left(\mathrm{~A}_{\mathrm{n}}\right)$. Intuitively, the correspondence comes from a "mirror" Schlegel diagram for an $n$-simplex, where the facets are reflected outwards from the hyperplane of projection.

Example 4.9. The fan $P_{1}\left(\mathrm{~A}_{\mathrm{n}}\right)$ is simplicial, with the Stanley-Reisner ring $\mathbb{Z}\left[x_{0}, \ldots, x_{n}\right] /\left\langle x_{0} \cdots x_{n}\right\rangle$. This is easily seen to have Hilbert series

$$
\frac{1-t^{n+1}}{(1-t)^{n+1}}=\sum_{i=0}^{n} t^{i} /(1-t)^{n}
$$

which is indeed equal to the Hilbert series of $D\left(\mathrm{~A}_{\mathrm{n}}\right) \simeq \bigoplus_{i=0}^{n} S(-i)$. By Corollary 4.7, this is also equal to $H S\left(A_{T}^{*}\left(X_{P_{2}\left(\mathrm{~A}_{\mathrm{n}}\right)}\right)_{\mathbb{Q}}, t\right)$.

Question 4.10. Is it possible to extend Theorem 4.6 to other classical groups? Types B and D have equations of the form $\left(x_{i} \pm x_{j}\right)$, so it is not obvious how to make such an extension. Computational evidence indicates a generalization might be possible for deformations of type A arrangements.

\section{ACKNOWLEDGMENTS}

Macaulay2 computations were essential to our work. The author thanks Michael DiPasquale, Sam Payne and Vic Reiner for useful conversations.

\section{REFERENCES}

[1] E. Bifet, C. De Concini, C. Procesi, Cohomology of regular embeddings, Advances in Mathematics 82 (1990), 1-34. MR 1057441 (91h:14052)

[2] L. Billera. The algebra of continuous piecewise polynomials, Advances in Mathematics 76 (1989), 170-183. MR1013666 (90g:13021)

[3] L. Billera and L. Rose, A dimension series for multivariate splines, Discrete and Computational Geometry 6 (1991), 107-128. MR1083627 (92g:41010)

[4] M. Brion, Piecewise polynomial functions, convex polytopes and enumerative geometry, Parameter spaces, 25-44, Banach Center Pub 36, Warsaw, 1996. MR.1481477 (99h:14052)

[5] M. Brion, Equivariant Chow groups for torus actions, Transform. Groups 2 (1997), 225-267. MR1466694 (99c:14005)

[6] M. Brion, M. Vergne, An equivariant Riemann-Roch theorem for complete, simplicial toric varieties, J. Reine Angew. Math. 482 (1997), 67-92. MR.1427657 (98a:14067)

[7] W. Bruns, J. Herzog, Cohen-Macaulay rings, Cambridge University Press, Cambridge, 1993. MR:1251956 (95h:13020)

[8] T. Mcdonald, H. Schenck, Piecewise polynomials on polyhedral complexes, Advances in Applied Mathematics, 42 (2009), 82-93. MR2475315 (2009k:41013)

[9] S. Payne, Equivariant Chow cohomology of toric varieties, Math. Res. Lett. 13 (2006), 29-41. MR2199564 (2007f:14052)

[10] H. Schenck, A spectral sequence for splines, Adv. Appl. Math. 19 (1997), 183-199. MR 1459497 (98i:13031) 
[11] H. Terao, Generalized exponents of a free arrangement of hyperplanes and Shepard-ToddBrieskorn formula, Invent. Math. 63 (1981), 159-179. MR608532 (82e:32018b)

[12] H. Terao, Free arrangements of hyperplanes and unitary reflection groups, Proc. Japan Acad. Ser. A 56 (1980), 389-392. MR.596011 (82e:32018a)

Department of Mathematics, University of Illinois Urbana-Champaign, Urbana, IlliNOIS 61801

E-mail address: schenck@math.uiuc.edu 